\title{
Molecular analysis of the determination of developmental fate in the small intestinal epithelium in the chicken embryo
}

\author{
HIROAKI HIRAMATSU and SADAO YASUGI* \\ Department of Biological Sciences, Graduate School of Science, Tokyo Metropolitan University, Tokyo, Japan.
}

\begin{abstract}
Determination of the developmental fate in the small intestinal epithelium of the chicken embryo has not been fully analyzed up to the present. This study was carried out to analyze the determination time of the developmental fate of the small intestinal epithelium under the influence of other mesenchymes. The small intestinal epithelium reassociated and cultivated with the proventricular or gizzard mesenchyme or the dermis expressed chicken intestinal fatty acid binding protein, sucrase and CdxA as occurs during the normal development of the small intestinal epithelium. The presumptive intestinal endoderm taken from an earlier stage embryo and associated and cultivated with the proventricular or gizzard mesenchyme, showed gene expression patterns which were the same as those found in normal development. However, when the dermis was associated, the epithelium expressed sonic hedgehog, but never expressed intestinal epithelial- or stomach epithelial-markers. These results indicate that the determination of the developmental fate in the small intestinal epithelium and acquisition of autodifferentiation potency occur at the early stage of the gut development. Moreover the presumptive intestinal endoderm needs the supportive influence of the gut mesenchyme in order to differentiate fully into the intestinal epithelium.
\end{abstract}

KEY WORDS: chicken, small intestinal epithelium, cIFABP, autodifferentiation potency

\section{Introduction}

The digestive organs of vertebrates consist of the epithelium derived from the endoderm and the mesenchyme derived from the splanchnic mesoderm. In chicken embryos, the right and left splanchnopleures fuse in the midline, and form a tube on day 2 to 3 of incubation. At this time, the epithelium is posted inside and the mesenchyme outside. On day 5, the digestive tract becomes divided anteropostriorly into the esophagus, proventriculus (glandular stomach), gizzard (muscular stomach), duodenum, small intestine, cecum, large intestine and allantois. Thereafter both the epithelium and the mesenchyme of each organ gradually differentiate and acquire organ specific characteristics. The esophageal epithelium becomes stratified epithelium with mucus glands. The proventricular epithelium invaginates into the mesenchymal tissue to form the glandular structures and gland epithelial cells begin to express ECPg (embryonic chicken pepsinogen; Hayashi et al., 1988a, b), a zymogen of digestive enzyme pepsin, from day 9 of incubation. The gizzard epithelium forms the simple glands and secretes mucus. The epithelia of these organs situated in the anterior part of the digestive tract commonly express a transcription factor cSox2 (Ishii et al., 1998) and a trefoil factor cSP (chicken spasmolytic polypeptide; Tabata and Yasugi, 1998). The epithelia of the small intestine and large intestine form villous structures and secrete some digestive enzymes such as sucrase.

The importance of the mesenchyme in the development of the proventricular epithelium and gizzard epithelium was repeatedly shown by the experimental studies (Mizuno and Yasugi, 1990; Yasugi, 1994; Yasugi and Fukuda, 2000). For example, when the gizzard epithelium of 6 day embryo was recombined and cultivated with the proventricular mesenchyme of 6 day embryo, the epithelium formed gland structures and expressed ECPg, suggesting that the gizzard epithelium differentiated into the proventricular epithelium (Takiguchi et al., 1986; Hayashi et al., 1988b; Urase et al., 1996). The factors responsible for the induction by the proventricular mesenchyme have been identified as extracellular matrices (Koike and Yasugi, 1999) and BMP-2 (Narita et al., 2000).

Abbreviations used in this paper: $\mathrm{CSP}$, chicken spasmolytic polypeptide; ECPg, embryonic chicken pepsinogen; IFABP, intestinal fatty acid binding protein.

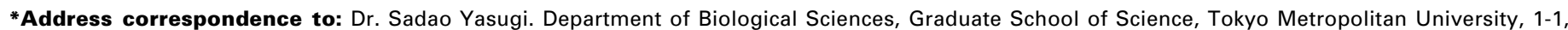
Minamiohsawa, Hachioji, Tokyo, 192-0397, Japan. Fax: +81-426-77-2572. e-mail: sadao-yasugi@c.metro-u.ac.jp
} 
On the other hand, the small intestinal epithelium of 6 day embryo never produced ECPg even under the influence of the proventricular mesenchyme and invariably expressed sucrase, a marker of intestinal epithelial cells (Yasugi et al., 1985; Hayashi et al., 1988b; Urase et al., 1996). It is therefore suggested that the potency to react to the induction of the proventricular mesenchyme is different in the small intestinal epithelium from that in the proventricular or gizzard epithelium of 6 day embryo. Moreover, when the presumptive intestinal endoderm from 1-2 day embryos was recombined and cultivated with the proventricular mesenchyme of 6 day embryo, the epithelium did not express ECPg (Yasugi etal., 1991). These results indicate that the small intestinal epithelium loses the responsiveness to mesenchymal induction early in the development. Moreover, the intestinal epithelium taken from 2.5 to 9 day embryos, wrapped with vitelline membrane and cultivated alone, could autodifferentiate into mature intestinal epithelium with PAS-positive goblet cells (Sumiya, 1976).

In these studies mentioned above, the digestive enzyme sucrase and some morphological criteria were used as the differentiation marker of the small intestinal epithelium. Also, the expression of $C d x A$, a homeobox gene specifically expressed in the intestinal epithelium, was examined in the presumptive intestinal endoderm recombined with the proventricular mesenchyme (Ishii et al., 1997). We have also studied the expression of $C S P$ (Tabata and Yasugi, 1998) and cSox2 (Ishii et al., 1998) in the small intestinal epithelium recombined with the proventricular mesenchyme, but the data are still fragmentary. At present many other genes are known to be expressed specifically in the small intestinal epithelium. Among them, a gene encoding an intestinal fatty acid binding protein (IFABP), a low molecular weight cysteine rich protein that is involved in the cellular uptake of fatty acids as well as their transport within the cell to particular organelles (Sweetser et al., 1987), is abundantly expressed in the small intestinal epithelium in zebrafish and Xenopus. However, its expression in the small intestinal epithelium associated with heterologous mesenchyme has not been examined. Horb and Slack (2001), using IFABP as a differentiation marker, showed that the endoderm from Xenopus neurula and tail-bud-stage possesses considerable ability to respond to the action of mesoderm from various regions.

In this study, we analyzed precisely and comprehensively the determination period of developmental fate of the small intestinal epithelium with epithelial-mesenchymal interactions, by cultivating tissue recombinants of the (presumptive) small intestinal epithelium and the mesenchymes from other digestive organs. Also we examined the ability of autodifferentiation in the small intestinal epithelium or presumptive intestinal endoderm by recombining them with dorsal skin dermis. The results clearly indicate that the developmental fate of the small intestinal epithelium is determined very early in the development, and that the realization of fully differentiated state requires some signals provided by the digestive organ mesenchymes.

\section{Results}

\section{Expression patterns of small intestinal epithelial markers during development}

To examine the expression pattern of $C / F A B P$, we dissected the small intestine of embryos of various developmental stages and examined it with in situ hybridization. The expression of CIFABP was not detected before day 6 (Fig. $1 \mathrm{~B}$ ), and began to be detected at day 7 of incubation (Fig. 1C), and was maintained during embryonic period (Fig. $1 \mathrm{D}, \mathrm{E})$. The expression of $c / F A B P$ was seen specifically in the small intestinal epithelium, not in the esophagus, proventriculus and gizzard (data not shown). We also re-investigated the expression of sucrase by the immunohistochemical technique and of $C d x A$ by in situ hybridization technique. As is reported previously (Matsushita, 1985), sucrase was first expressed in 10 day embryo and was maintained after this stage up to post-hatch stages except that it disappeared just before hatching (Fig. $1 \mathrm{~F}, \mathrm{G}$ ). Expression pattern of $C d x A$ in the chicken embryo was previously reported (Ishii et al., 1997). Expression begins in the gut endoderm at stage 10 (Hamburger and Hamilton, 1951), and continues throughout the embryonic stage (Fig. $1 \mathrm{H}-J$ ). Thus $c / F A B P$, as sucrase and $C d x A$, is a specific marker of the small intestinal epithelium.

\section{Reactivity of the small intestinal epithelium of 6 day embryo to 6 day proventricular mesenchyme or gizzard mesenchyme}

We first carried out tissue recombination experiments using digestive organs of 6 day embryo to confirm that the developmental fate of the small intestinal epithelium in the chicken embryo is already determined at day 6 of incubation. When the epithelium and mesenchyme of the same organ were recombined, expres-
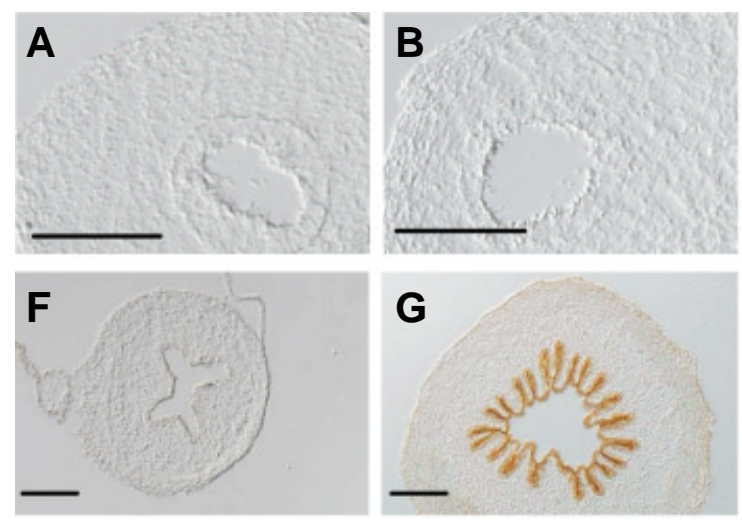
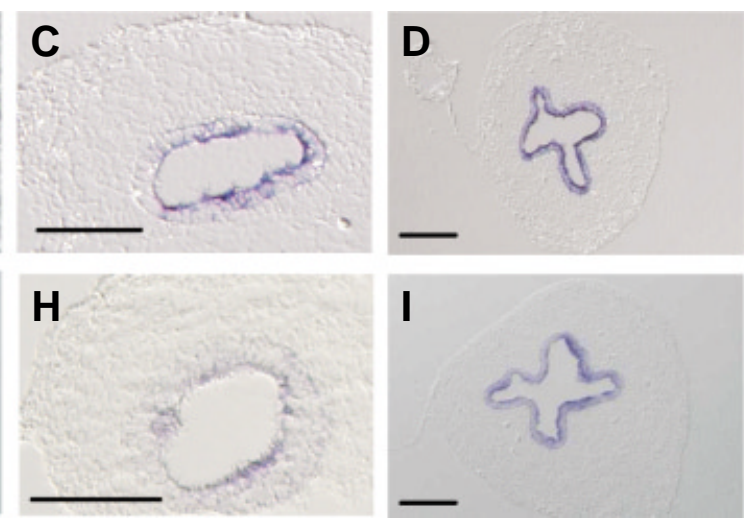
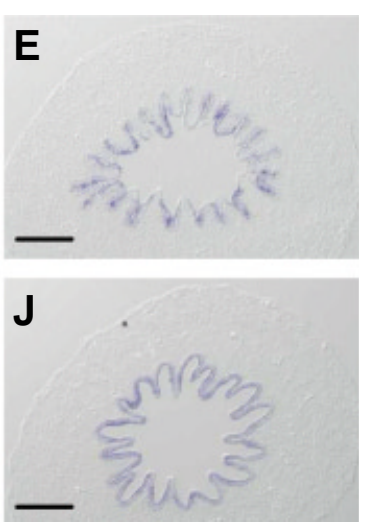

Fig. 1. Expression patterns of $\operatorname{cIFABP}(\mathbf{A}-\mathbf{E})$, sucrase $(\mathbf{F}, \mathbf{G})$ and $\boldsymbol{C d x} \boldsymbol{A}(\mathbf{H}-\mathbf{J})$ in the small intestine $(A-J)$, of $5(A), 6(B, H), 7(C), 9(D, F)$ and $12(E, G, J)$ day embryos analyzed in transverse sections by in situ hybridization (cIFABP and CdxA) or immunohistochemistry (sucrase). Scale bars, 100 um. 

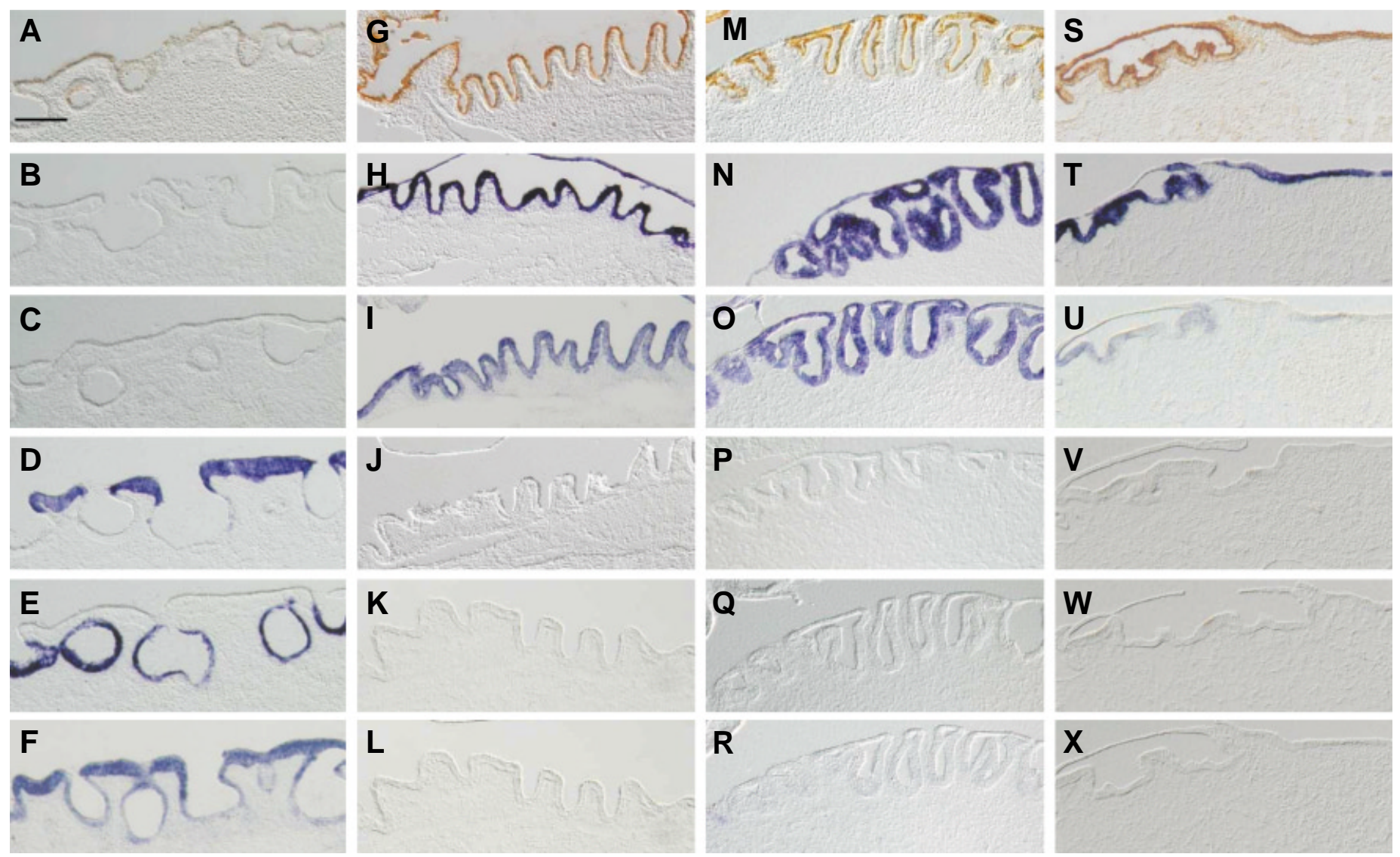

Fig. 2. Expression of sucrase, $C I F A B P, C d x A, C S P, E C P g$ and $c S o x 2$ in the recombinants of small intestinal epithelium associated with heterologous gut mesenchymes. The proventricular epithelium of 6 day embryo cultivated with 6 day proventricular mesenchyme (A-F). The small intestinal epithelium of 6 day embryo cultivated with 6 day small intestinal mesenchyme (G-L), proventricular mesenchyme (M-R) and gizzard mesenchyme $(S-X)$. Expression of sucrase $(\mathbf{A}, \mathbf{G}, \mathbf{M}, \mathbf{S})$, cIFABP $(\mathbf{B}, \mathbf{H}, \mathbf{N}, \mathbf{T}), \operatorname{CdxA}(\mathbf{C}, \mathbf{I}, \mathbf{O}, \mathbf{U}), \operatorname{cSP}(\mathbf{D}, \mathbf{J}, \mathbf{P}, \mathbf{V}), \mathrm{ECPg}(\mathbf{E}, \mathbf{K}, \mathbf{Q}, \mathbf{W})$ and cSox2 $(\mathbf{F}, \mathbf{L}, \mathbf{R}, \mathbf{X})$ was analyzed by immunohistochemistry or in situ hybridization in adjacent sections. Scale bar, $100 \mu \mathrm{m}$.

sions of marker molecules were the same as in normal development (Fig. 2 A-L, Table 1). In brief, when the proventricular epithelium was cultivated with proventricular mesenchyme, the explants expressed $C S P$ in luminal epithelium, ECPgin glandular epithelium and $C$ Sox2in both luminal and glandular epithelia (Fig. $2 \mathrm{D}-\mathrm{F})$. When the small intestinal epithelium was cultivated with the small intestinal mesenchyme, the epithelium expressed su-

\section{TABLE 1}

\section{GENE EXPRESSION IN RECOMBINANTS COMPOSED OF EPITHELIUM AND 6 DAY MESENCHYME OF THE GUT}

\begin{tabular}{|c|c|c|c|c|c|c|c|c|}
\hline \multirow{2}{*}{ Epithelium ${ }^{c}$} & \multirow{2}{*}{ Mesenchyme $^{c}$} & \multirow{2}{*}{$\begin{array}{l}\text { Number of } \\
\text { recombinants }\end{array}$} & \multicolumn{6}{|c|}{ Expression ${ }^{a}$} \\
\hline & & & sucrase & cIFABP & CdxA & $\mathrm{cSP}$ & $\mathrm{ECPg}$ & cSox2 \\
\hline PV & PV & 24 & 0 & 0 & 0 & 100 & 100 & 100 \\
\hline SI & PV & 20 & 100 & 100 & 100 & 0 & 0 & 0 \\
\hline SI & $\mathrm{GZ}$ & 19 & 100 & 100 & 100 & 0 & 0 & 0 \\
\hline SI & SI & 20 & 100 & 100 & 100 & 0 & 0 & 0 \\
\hline PIE & PV & 12 & 0 & $100^{b}$ & $100^{b}$ & $92^{b}$ & 0 & 0 \\
\hline PIE & $\mathrm{GZ}$ & 16 & 0 & 0 & 0 & $94^{b}$ & 0 & 0 \\
\hline PIE & SI & 12 & 100 & 100 & 100 & 0 & 0 & 0 \\
\hline
\end{tabular}

a Percentages of recombinants in which expression was detected.

${ }^{b}$ Strong expression was seen partially in the epithelium.

c PV, proventriculus; GZ, gizzard; SI, small intestine; PIE, presumptive intestinal endoderm crase, $C / F A B P$ and $C d x A$ (Fig. 2 G-I) and genes specific to stomach epithelium were not expressed (Fig. $2 \mathrm{~J}-\mathrm{L}$ ).

When the small intestinal epithelium was cultured with the proventricular mesenchyme, the epithelium invaginated into the mesenchyme and showed the proventricular gland-like structures. However the epithelium of the explants expressed sucrase, CIFABPand $C d x A$, markers of the small intestinal epithelium (Fig. $2 \mathrm{M}-\mathrm{O}$ ), and never expressed $C S P, E C P g$ and $C S O x 2$, markers of stomach epithelium (Fig. 2 P-R). Moreover, the explants composed of the small intestinal epithelium and gizzard mesenchyme expressed markers of small intestinal epithelium (Fig. $2 \mathrm{~S}-\mathrm{U}$ ), but not $c S P$ and $C S O x 2$ (Fig. $2 \mathrm{~V}, \mathrm{X}$ ). Thus the intestinal epithelium of 6 day embryo was proved to be determined with its developmental fate and showed sharp contrast to the epithelia of the proventriculus and gizzard that retain reactivity to the inductive influence of the mesenchyme (Takiguchi et al., 1986; Hayashi et al., 1988b; Urase et al., 1996).

\section{Reactivity of the small intestinal epithelium of 6 day embryo to 6 day dermis}

Next, we tested the autodifferentiation potency of the small intestinal epithelium of 6 day embryo associated with dermis of the dorsal skin of 6 day embryo, which is supposed to have no activity to induce the epithelium of the digestive organs (Table 2). The 

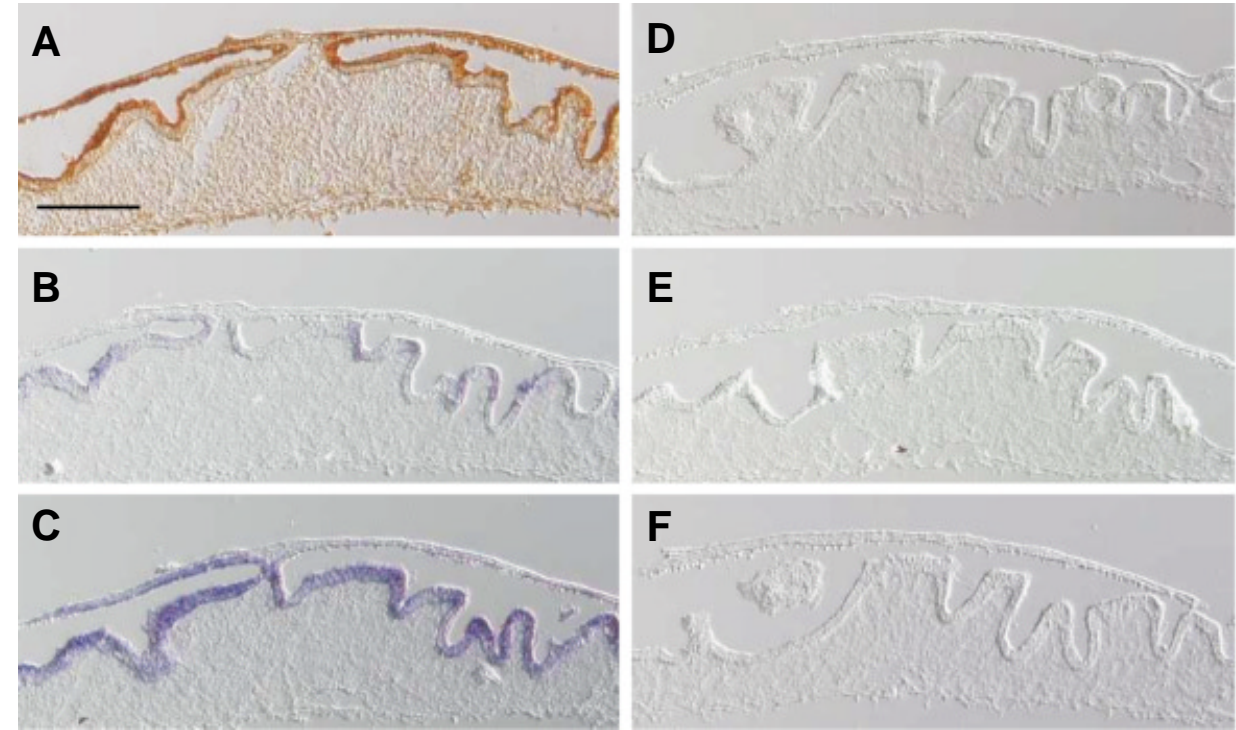

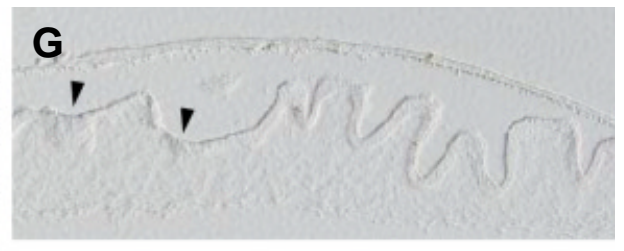

epithelium of explants strongly expressed sucrase, $C d x A$ and $c / F A B P$ (Fig. 3 A-C). Some cells of the epithelium expressed shh(Fig. 3G, arrowhead), a pan-endodermal marker. The explants did not show expression of $C S P, E C P g$ and $c S O x 2$ (Fig. $3 \mathrm{D}-\mathrm{F}$ ). The results clearly indicated that the small intestinal epithelium can autodifferentiate according to its own developmental fate.

Reactivity of the presumptive intestinal endoderm of 1.5 day embryo to 6 day proventricular mesenchyme or gizzard mesenchyme

The fact that the developmental fate of the small intestinal epithelium is already determined by day 6 of incubation raises the question whether the presumptive intestinal endoderm associated and cultivated with 6 day proventricular mesenchyme or gizzard mesenchyme can differentiate heterologously. When the presumptive intestinal endoderm of 1.5 day embryos was cultivated with small intestinal mesenchyme, the epithelium expressed sucrase, $C d x A$ and $C I F A B P$, markers of small intestinal epithelium (Fig. $4 \mathrm{~A}-\mathrm{C}$ ), but never expressed $c S P$, ECPgand $c S o x 2$ (Fig. $4 \mathrm{D}-\mathrm{F}$ ). The presumptive intestinal endoderm associated and cultivated with 6 day proventricular mesenchyme invaginated into the mesenchyme and formed the gland-like structures, but still expressed markers specific to the small intestinal epithelium except sucrase (Fig. 4 G-I). Among the markers of stomach epithelium, $C S P$ was expressed occasionally in small parts of the epithelium where intestinal markers were not expressed (Fig. 4J), but ECPgand cSox2could not be detected (Fig.

\section{TABLE 2}

\section{GENE EXPRESSION IN RECOMBINANTS COMPOSED OF EPITHE-} LIUM AND 6 DAY DORSAL DERMIS

\begin{tabular}{|c|c|c|c|c|c|c|c|c|}
\hline \multirow[b]{2}{*}{ Epithelium $^{d}$} & \multirow{2}{*}{$\begin{array}{l}\text { Number of } \\
\text { recombinants }\end{array}$} & \multicolumn{7}{|c|}{ Expression $^{a}$} \\
\hline & & sucrase & CIFABP & CdxA & shh & cSP & $\mathrm{ECPg}$ & cSox2 \\
\hline SI & 16 & 94 & 94 & 94 & $69^{b}$ & 0 & 0 & 0 \\
\hline PIE & 6 & 0 & 0 & 0 & $80^{c}$ & 0 & 0 & 0 \\
\hline
\end{tabular}

a Percentages of recombinants in which expression was detected.

${ }^{b}$ Expression was seen in very narrow areas of recombinants.

${ }^{c}$ Strong expression was seen partially in the epithelium.

d SI, small intestine; PIE, presumptive intestinal endoderm
$4 \mathrm{~K}, \mathrm{~L})$. When the presumptive intestinal endoderm was cultivated with 6 day gizzard mesenchyme, the epithelium expressed $C S P$ (Fig. 4P), marker of luminal epithelium of the stomach, but other stomach epithelial markers (Fig. $4 \mathrm{Q}, \mathrm{R}$ ) and markers of small intestinal epithelium were never expressed (Fig. $4 \mathrm{M}-\mathrm{O}$ ). These results indicated that the presumptive intestinal endoderm of 1.5 day embryo had different reactivity to the proventricular mesenchyme and gizzard mesenchyme.

\section{Reactivity of the presumptive intestinal endoderm of 1.5 day embryo to 6 day dermis}

Finally, we tested the autodifferentiation potency of the presumptive intestinal endoderm of 1.5 day embryo associated with 6 day dorsal dermis. The epithelium expressed shh (Fig. 5D), a panendodermal marker, suggesting it acquired the characteristics of endodermal epithelium, but never expressed other markers of the small intestinal epithelium and stomach epithelium (Fig. 5 A-C, Table 2). The endoderm never formed feather-like structures on the dorsal dermis.

The above results indicate that the developmental fate of the presumptive intestinal endoderm is almost decided before 1.5 day of embryonic development, and the presumptive intestinal endoderm differentiates into the small intestinal epithelium under the existence of the mesenchyme derived from splanchnic mesoderm.

\section{Discussion}

It has been repeatedly confirmed that the proventricular or gizzard epithelium associated with the mesenchyme of heterologous organs differentiates heterotypically according to the mesenchymal induction (Takiguchi et al., 1986; Hayashi et al., 1988b; Urase etal., 1996). On the other hand, the small intestinal epithelium of 6 day embryo, or even younger presumptive intestinal endoderm, associated and cultivated with the proventricular mesenchyme, never differentiated into ECPg-expressing epithelium (Urase et al., 1993; Yasugi et al., 1985; 1991). These results indicate that the (presumptive) small intestinal epithelium is determined as regards its developmental fate early in the development. In this study we analyzed more precisely and comprehensively the determination 
period of developmental fate of the small intestinal epithelium under epithelial-mesenchymal interactions with using the molecular differentiation markers, and investigated the autodifferentiation potency of the small intestinal epithelium. Among these markers, $C d x A$ is abundantly expressed in the small intestinal epithelium from early stages of development (Ishii etal., 1997). On the other hand, c/FABP and sucrase are expressed when the organ acquires its characteristic features (this study and Matsushita, 1985). Therefore these genes are useful markers of small intestinal epithelium at various stages of the development. It was reported that IFABP is expressed specifically in the small intestinal epithelium from early in the development in Xenopus (Chalmers and Slack, 1998) and that, during metamorphosis, IFABPexpression decreases as the tadpole intes- tinal epithelium is shed. As the more complex adult-like epithelium forms after metamorphic climax, IFABPexpression is restored (Shi and Par Hayes, 1994; Ishizuya-Oka et al., 1997). In this report we described developmental expression pattern of $c / F A B P$ for the first time. In chicken embryo, the expression of $c / F A B P$ begins on day 7 and continues thereafter, solely in the small intestinal epithelium.

\section{Reactivity of the 6 day small intestinal epithelium and 1.5 day} presumptive intestinal endoderm to heterologous mesenchymes

We tested whether the small intestinal epithelium, associated and cultivated with the proventricular mesenchyme or gizzard mesenchyme, expresses various intestinal marker genes. The morphological differentiation of the small intestinal epithelium
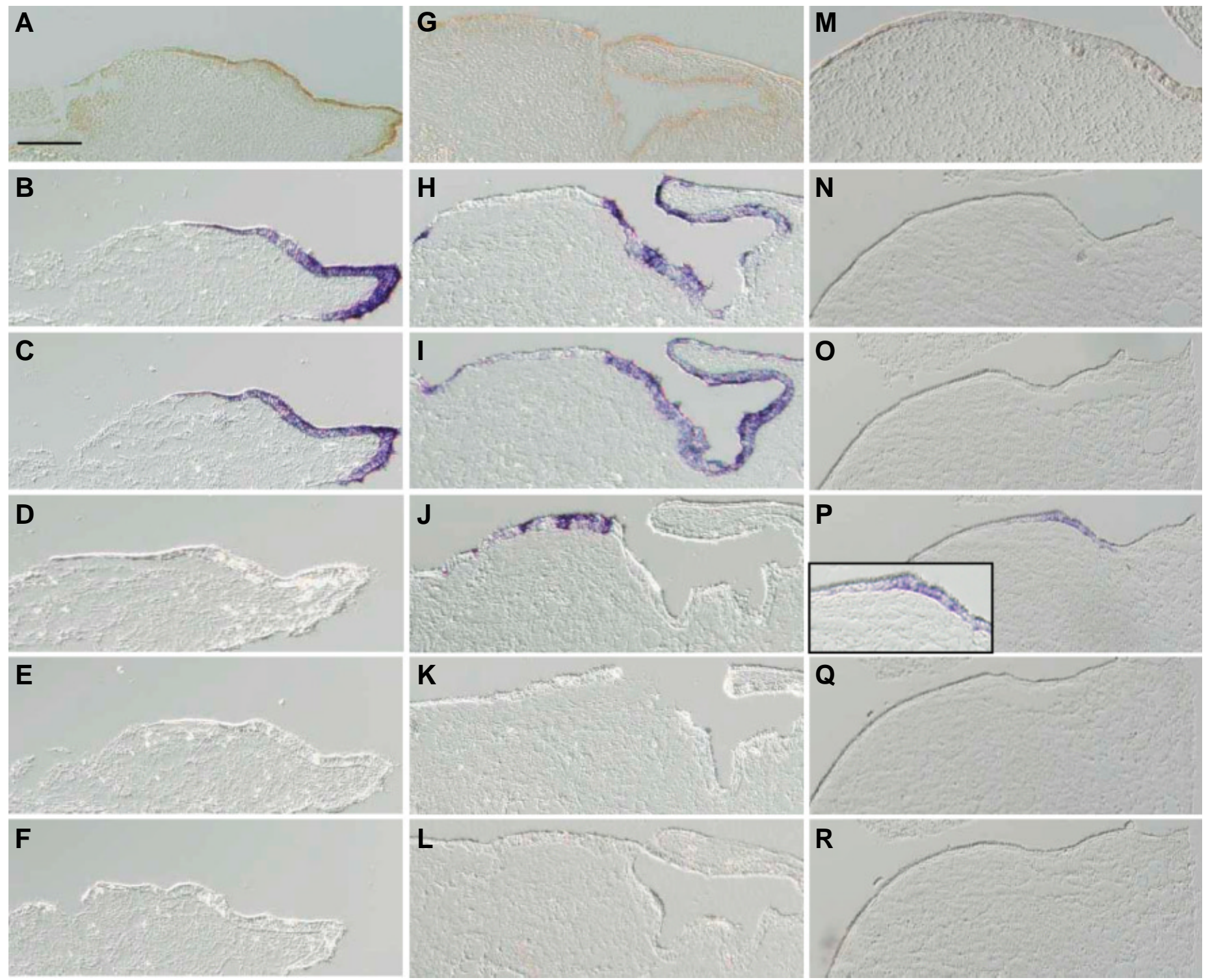

Fig. 4 (Left). Expression of sucrase, cIFABP, CdxA, $C S P, E C P g$ and $c$ Sox2 in the recombinants of presumptive intestinal endoderm associated with heterologous gut mesenchymes. The presumptive intestinal endoderm of 1.5 day embryo cultivated with 6 day small intestinal mesenchyme (A-F), proventricular mesenchyme (G-L) and gizzard mesenchyme (M-R). Expression of sucrase $(A, G, M), \operatorname{clFABP}(B, H, N), C d x A(C, I, O), c S P(D, J, P), E C P g$ $(E, K, Q)$ and cSox2 $(F, L, R)$ was analyzed by immunohistochemistry or in situ hybridization in adjacent sections. Inset in $P$ is a higher magnification view of the corresponding section. Scale bar, $100 \mu \mathrm{m}$. 


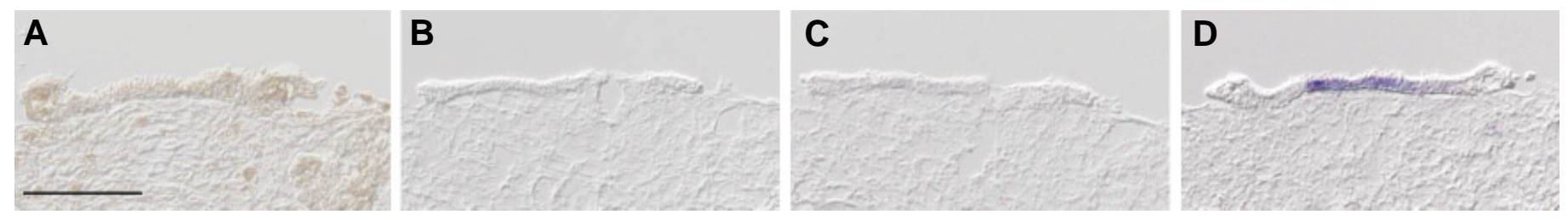

Fig. 5. Expression of sucrase (A), cIFABP (B), CdxA (C) and shh (D) in presumptive intestinal endoderm of 1.5 day embryo cultivated with 6 day dorsal skin dermis. Marker expression was analyzed by immunohistochemistry or in situ hybridization in adjacent sections. Scale bar, $100 \mu$ m.

depended on the nature of the mesenchyme associated: the epithelium formed gland like structures when it was associated with proventricular mesenchyme. However it invariably expressed sucrase, $C I F A B P$ and $C d x A$ and never expressed the stomach epithelial markers, suggesting that the influences of mesenchymal induction can occasionally affect morphological differentiation but not cytodifferentiation.

Presumptive intestinal endoderm of 1.5 day embryo showed different reactivity to the proventricular mesenchyme and gizzard mesenchyme of 6 day embryo. In the association with the proventricular mesenchyme, the endoderm expressed $C / F A B P$ and $C d x A$, while these genes were not expressed in the association with the gizzard mesenchyme. In both cases $C S P$, marker of stomach epithelium, was induced in some parts of the epithelium. This difference in reactivity may attributable to the difference in the inductive ability of two mesenchymes: the gizzard mesenchyme exerts stronger influence on the heterotypic differentiation of the small intestinal (duodenal) epithelium than the proventricular mesenchyme (Matsushita, 1995b). Thus the presumptive intestinal endoderm, though the main developmental fate is already determined at 1.5 day, could respond to the action of the proventricular and gizzard mesenchymes and express $c S P$, it did not express intestinal markers under the influence of the gizzard mesenchyme.

In both combinations, sucrase was scarcely detected in the presumptive intestinal endoderm. It has been reported that $C d \times 2$ regulates the expression of sucrase in the mouse (Lorentz et al., 1997) and that $C d x A$ binds to the promoter region of sucrase (Sklan et al., 2003). It is therefore possible that $C d x A$ regulates the expression of sucrase in the chicken. When the presumptive intestinal endoderm was associated with the proventricular mesenchyme, $C d x A$ was widely expressed but there was no or only weak expression of sucrase. This may due partly to the cultivation period of the recombinants. We cultivated recombinants for 6 days so that they corresponded to embryos of 7.5 or 8 day of incubation. At this stage sucrase was not yet expressed in the normal course of development.

\section{Autodifferentiation potency of 6 day small intestinal epithe- lium and 1.5 day presumptive intestinal endoderm}

When the small intestinal epithelium associated with dorsal skin dermis which is supposed to have no activity to induce the epithelium of the digestive organs, the epithelium expressed sucrase, $C I F A B P$ and $C d x A$, but never expressed stomach epithelial markers. From the results, we thought that the small intestinal epithelium has lost reactivity to other heterologous mesenchymes, and it has an autodifferentiation potency. This result is different from the report that, when the proventricular epithelium was associated with the dorsal dermis, it never expressed ECPg and the characteristics of the small intestinal epithelium was observed (Urase et al., 1996).

On the other hand, when the presumptive intestinal endoderm of 1.5 day embryo associated with dorsal skin dermis, the epithelium never expressed markers of small intestinal epithelium and stomach epithelium. The fact that expression of shh, a panendodermal marker, was maintained in the epithelium indicates that the developmental fate of younger presumptive intestinal endoderm was almost determined but the influences of the mesenchymes of the digestive organs are necessary for the differentiation of the small intestinal epithelium. These results are consistent with the report that the lateral plate mesoderm has instructive influence on the formation of dorsal pancreas (Kumar et al., 2003). Also in mammals, it was demonstrated that presumptive intestinal endoderm is determined according to its origin as for its differentiation fate (Duluc et al., 1994; Ratineau et al., 2003).

\section{Molecular mechanism of regionalization in gut development}

Our present results and many reports lead to the conclusion that developmental fate of the posterior gut endoderm is determined early in the development while the anterior gut endoderm seems to retain the reactivity to the heterologous mesenchymes up to day 6 of incubation. The molecular mechanisms underlying the regionalization in gut development has not been clearly understood. We suppose that $C d x A$ is involved as one of determination factors. $C d x A$ gene is specifically expressed in the endoderm which becomes midgut and hindgut before 1.5 day (Ishii et al., 1997). Moreover, it was reported that mouse $C d \times 1$ and $C d x 2$ are important in anterior-posterior patterning in the intestinal epithelium (Silberg et al., 2000). Cdx2 defines the stomachintestine boundary and when the function of $C d \times 2$ was downregulated, the proximal part of the intestine formed the gastrictype tissue (Beck et al., 2003). From these reports, $C d x A$ is thought to be an important factor involved in the determination of the developmental fate of the small intestinal epithelium in the chicken embryo. We are currently investigating the effects of ectopic expression of $C d x A$ gene in the presumptive stomach endoderm of young embryo.

\section{Materials and Methods}

\section{Animals}

Embryos of White Leghorn chickens (Gallus gallus domesticus) were used throughout the experiments.

\section{Isolation of tissue fragments}

The presumptive intestinal endoderm was obtained from embryos of 1.5 days of incubation (embryos with 15 to 20 somites, stage 10-12, 
Hamburger and Hamilton, 1951). At this stage, the presumptive stomach endoderm locates just under the notochord of level of somite 2-7, and the presumptive intestinal endoderm locates more caudally and laterally (Matsushita, 1995a; 1996; 1999). We used the endoderm of level of somite 10-15 of embryos as a source of presumptive intestinal endoderm. The endodermal fragments were isolated from notochord, somites and lateral plate mesoderm by mild treatment of collagenase type I (Worthington Biochemical Corporation, Lakewood, NJ; Code CLS1, 0.03\% in Tyrode's solution for $15 \mathrm{~min}$ at $38^{\circ} \mathrm{C}$ ). Epithelial fragments and the mesenchymes of the proventriculus, gizzard and small intestine (a part just anterior to the yolk sac stalk) of 6 day embryos were obtained by longer (30-60 min) treatment of collagenase. The epithelia were stripped off as a sheet from the underlying mesenchymes and both tissues were washed in Tyrode's solution. The dorsal dermis was isolated from the dorsal skin of 6 day embryos. The skin fragments were rinsed in a calcium- and magnesiumfree (CMF) Tyrode's solution, and then treated with double-strength CMF Tyrode's solution containing disodium ethylenediamine tetraacetate (EDTA, $0.25 \%$ ) at $20^{\circ} \mathrm{C}$ for 1 to 3 hours (Mizuno et al., 1990; Urase et al., 1996). The epidermis were stripped off as a sheet from the underlying dermis and washed in Tyrode's solution.

\section{Tissue recombination experiments and in vitro cultivation tech- nique}

Epithelium or presumptive intestinal endoderm was reassociated with the mesenchyme on the semi-solid agar substratum (Wolff and Haffen, 1952) for $12 \mathrm{~h}$ at $37^{\circ} \mathrm{C}$ to ensure the coherence of two tissue fragments. The agar substratum was composed of seven parts of agar (2\% agar in Gay's solution), 3 parts of Medium 199 with Earle's salts (Gibco, USA) and 3 parts of 13 day chicken digestive tract- and eye-free embryo extract. Recombinants were transferred onto Nuclepore filters (Whattman, USA) of pore size $0.8 \mu \mathrm{m}$, rested on stainless-steel grid which was placed within a small culture dish. The medium reached the Nuclepore filter and moistened surface of the explants but not covered them. The culture dishes were incubated for 6 days at $37^{\circ} \mathrm{C}$ in $95 \%$ air and $5 \% \mathrm{CO}_{2}$. The culture medium was Medium 199 with Earle's salts supplemented with $50 \% 13$ day embryo extract and penicillin $(100 \mathrm{U} / \mathrm{ml})$. The culture medium was replaced with flesh medium every second day (Takiguchi et al., 1988).

\section{In situ hybridization}

Digoxygenin-labeled RNA probes for in situ hybridization were prepared from cDNA clones of ECPg (Hayashi et al., 1988), CSP(Tabata et al., 1998), cSox2 (Uwanogho et al., 1995), sonic hedgehog (shh, Nohno et al., 1995), chicken IFABP (CIFABP, MRC Geneservice, Cambridge) and $C d x A$ (Ishii et al., 1997). Organs and explants were fixed with $4 \%$ paraformaldehyde in phosphate-buffered-saline (PBS) overnight at $4^{\circ} \mathrm{C}$ and embedded in OCT compound (Sakura Finetechnical Co. Ltd., Tokyo Japan). Frozen sections of $10 \mu \mathrm{m}$ were cut in a cryostat. In situhybridization on frozen sections was performed as previously described (Ishii et al., 1997).

\section{Immunohistochemistry}

Adjacent frozen sections were used for in situ hybridization and immunohistochemistry. After rehydration with PBS, sections were blocked with $0.5 \%$ skim milk in PBS and treated with polyclonal anti-chicken sucrase antibody (Matsushita, 1985) overnight at $4^{\circ} \mathrm{C}$. After three washes with PBS at room temperature, sections were treated with horse radish peroxidase-conjugated anti-rabbit immunoglobulin $\mathrm{G}$ for $1 \mathrm{~h}$ at $37^{\circ} \mathrm{C}$, and washed with PBS three times. Coloring reaction was done with $0.2 \mu \mathrm{g} / \mathrm{ml}$ diaminobenzidine in $0.1 \mathrm{M}$ Tris- $\mathrm{HCl}(\mathrm{pH} 7.5)$ and $0.001 \% \mathrm{H}_{2} \mathrm{O}_{2}$.

\section{Acknowledgements}

The authors thank Susumu Matsushita of the Tokyo Women's Medical College for kind gift of anti-sucrase antibody. This work was supported in part by Grant-in-Aid for Scientific research on Priority Areas (13044002) from the Ministry of Education, Culture, Sports, Science and Technology of Japan to S. Y. and by the Sasakawa Scientific Research Grant from The Japan Science Society to $H$. $H$.

\section{References}

BECK, F., CHAWENGSAKSOPHAK, K., LUCKETT, J., GIBLETT, S., TUCCI, J., BROWN, J., POULSOM, R., JEFFERY, R. and WRIGHT, N.A. (2003). A study of regional gut endoderm potency by analysis of $C d \times 2$ null mutant chimeric mice. Dev. Biol. 255: 399-406

CHALMERS, A.D. and SLACK, J.M. (1998). Development of the gut in Xenopus laevis. Dev. Dyn. 212: 509-521.

DULUC, I., FREUND, J.N., LEBERQUIER, C. and KEDINGER, M. (1994). Fetal endoderm primarily holds the temporal and positional information required for mammalian intestinal development. J. Cell Biol. 126: 211-221.

HAMBURGER, V. and HAMILTON, H.L. (1951). A series of normal stages in the development of the chicken embryo. J. Morph. 88: 49-92.

HAYASHI, K., AGATA, K., MOCHII, M., YASUGI, S., EGUCHI, G. and MIZUNO, T. (1988a). Molecular cloning and the nucleotide sequence of cDNA for embryonic chicken pepsinogen: phylogenetic relationship with prochymocin. J. Biochem. 103: 290-296.

HAYASHI, K., YASUGI, S. and MIZUNO, T. (1988b). Pepsinogen gene transcription induced in heterologous epithelial-mesenchymal recombinations of chicken endoderms and glandular stomach mesenchyme. Development 103: 725-731.

HORB, M.E. and SLACK, J.M.W. (2001). Endoderm specification and differentiation in Xenopus embryos. Dev. Biol. 236: 330-343.

ISHII, Y., FUKUDA, K., SAIGA, H., MATSUSHITA, S. and YASUGI, S. (1997). Early specification of intestinal epithelium in the chicken embryo: a study on the localization and regulation of CdxA expression. Dev. Growth Differ. 39: 643653.

ISHII, Y., REX, M., SCOTTING, P.J. and YASUGI, S. (1998). Region-specific expression of chicken Sox2 in the developing gut and lung epithelium: regulation by epithelial-mesenchymal interactions. Dev. Dyn. 213: 464-475.

ISHIZUYA-OKA, A., UEDA, S., DAMJANOVSKI, S., LI, Q., LIANG, V.C. and SHI Y.B. (1997). Anteroposterior gradient of epithelial transformation during amphibian intestinal remodeling: immunohistochemical detection of intestinal fatty acid-binding protein. Dev. Biol. 192: 149-161.

KOIKE, T. and YASUGI, S. (1999). In vitro analysis of mesenchymal influences on the differentiation of stomach epithelial cells of chicken embryo. Differentiation 65: 13-25.

KUMAR, M., JORDAN, N., MELTON, D. and GRAPIN-BOTTON, A. (2003). Signals from lateral plate mesoderm instruct endoderm toward a pancreatic fate. Dev. Biol. 259: 109-122.

LORENTZ, O., DULUC, I., DE ARCANGELIS, A., SIMON-ASSMANN, P., KEDINGER, M. And FREUND, J.N. (1997). Key role of the Cdx2 homeobox gene in extracellular matrix-mediated intestinal cell differentiation. J. Cell Biol. 139: $1553-1565$

MATSUSHITA, S. (1985). Development of sucrase in the chick small intestine. J. Exp. Zool. 233: 377-383.

MATSUSHITA, S. (1995a). Fate mapping study of the splanchnopleural mesoderm of the 1.5-day-old chick embryo. Roux's Arch. Dev. Biol. 204: 392-399.

MATSUSHITA, S. (1995b). Regional difference in the responsiveness to the inductive influence of the gizzard mesenchyme among the endoderms of various small intestinal segments of the chick embryo. Dev. Growth Differ. 37: 201-209.

MATSUSHITA, S. (1996). Fate mapping study of the endoderm of the 1.5-day-old chick embryo. Roux's Arch. Dev. Biol. 205: 225-231.

MATSUSHITA, S. (1999). Fate mapping study of the endoderm in the posterior part of the 1.5-day-old chick embryo. Dev. Growth Differ. 41: 313-319.

MIZUNO, T., KITAMURA, K., SAITO, M. and TANEMURA, S. (1990). Epithelial metaplasia induced on amnionic ectoderm by the dermis of chicken embryo. Dev. Growth Differ. 32: 171-177.

MIZUNO, T. and YASUGI, S. (1990). Susceptibility of epithelia to directive influences of mesenchymes during organogenesis: uncoupling of morphogenesis and cytodifferentiation. Cell Differ. Dev. 31: 151-159. 
NARITA, T., SAITOH, K., KAMEDA, T., KUROIWA, A., MIZUTANI, M., KOIKE, C., IBA, H. and YASUGI, S. (2000). BMPs are necessary for stomach gland formation in the chicken embryo: a study using virally induced BMP-2 and Noggin expression. Development 127: 981-988.

NOHNO, T., KAWAKAMI, Y., OHUCHI, H., FUJIWARA, A., YOSHIOKA, H. and NOJI, S. (1995). Involvement of the Sonic hedgehog gene in chick feather formation. Biochem. Biophys. Res. Commun. 206: 33-39.

RATINEAU, C., DULUC, I., POURREYRON, C., KEDINGER, M., FREUND, J. N. and ROCHE, C. (2003). Endoderm- and mesenchyme-dependent commitment of the differentiated epithelial cell types in the developing intestine of rat. Differentiation 71: 163-169.

SHI, Y.B. and PAR HAYES, W.P. (1994). Thyroid hormone-dependant regulation of the intestinal fatty acid-binding protein gene during amphibian metamorphosis. Dev. Biol. 161: 48-58.

SILBERG, D.G., SWAIN, G.P., SUH, E.R. and TRABER, P.G. (2000). Cdx1 and cdx2 expression during intestinal development. Gastroenterology 119: 961 971.

SKLAN, D., GEYRA, A., TAKO, E., GAL-GERBER, O. and UNI, Z. (2003). Ontogeny of brush border carbohydrate digestion and uptake in the chick. Br. J. Nutr. 89: 747-753

SUMIYA, M. (1976). Differentiation of the digestive tract epithelium of the chick embryo cultured in vitro enveloped in a fragment of the vitelline membrane, in the absence mesenchyme. Roux's Arch. Dev. Biol. 179: 1-17.

SWEETSER, D.A., HEUCKEROTH, R.O. and GORDON, J.I. (1987). The metabolic significance of mammalian fatty-acid-binding proteins: abundant proteins in search of a function. Annu. Rev. Nutr. 7: 337-359.

TABATA, H. and YASUGI, S. (1998). Tissue interaction regulates expression of a spasmolytic polypeptide gene in chicken stomach epithelium. Dev. Growth Differ. 40: 519-526.

TAKIGUCHI, K., YASUGI, S. and MIZUNO, T. (1986). Gizzard epithelium of chick embryos can express embryonic pepsinogen antigen, a marker protein of proventriculus. Roux's Arch. Dev. Biol. 195: 475-483.
TAKIGUCHI, K., YASUGI, S. and MIZUNO, T. (1988). Pepsinogen induction in chick stomach epithelia by reaggregated proventricular mesenchyme cells in vitro. Dev. Growth Differ. 30: 241-250.

URASE, K. and YASUGI, S (1993). Induction and inhibition of epithelial differentiation by the mixed cell aggregates of the mesenchymes from the chicken embryonic digestive tract. Dev. Growth Differ. 35: 33-40.

URASE, K., FUKUDA, K., ISHII, Y., Sakamoto, N. and Yasugi, S. (1996). Analysis of mesenchymal influence on the pepsinogen gene expression in the epithelium of chicken embryonic digestive tract. Roux's Arch. Dev. Biol. 205: 382-390.

UWANOGHO, D., REX, M., CARTWRIGHT, E.J., PEARL, G., HEALY, C., SCOTTING, P.J. and SHARPE, P.T. (1995). Embryonic expression of the chicken Sox2, Sox3and Sox11genes suggests an interactive role in neuronal development. Mec. Dev. 49: 23-26.

WOLFF, E. and HAFFEN, K. (1952). Su rune méthode de culture d'organes embryonnaires "in vitro". Tex. Rep. Biol. Med. 10: 463-472.

YASUGI, S. (1994). Regulation of pepsinogen gene expression in epithelial cells of vertebrate stomach during development. Int. J. Dev. Biol. 38: 273-279.

YASUGI, S. and FUKUDA, K. (2000). The mesenchymal factors regulating epithelial morphogenesis and differentiation of the chicken stomach. Zool. Sci. 17: 1-9.

YASUGI, S., MATSUSHITA, S. and MIZUNO, T. (1985). Gland formation induced in the allantoic and small-intestinal endoderm by the proventricular mesenchyme is not coupled with pepsinogen expression. Differentiation 30: 47-52.

YASUGI, S., TAKEDA, H. and FUKUDA, K. (1991). Early determination of developmental fate in presumptive intestinal endoderm of the chicken embryo. Dev. Growth Differ. 33: 235-241.

Received: July 2004

Reviewed by Referees: August 2004

Modified by Authors and Accepted for Publication: September 2004 